\title{
Pharmacological and histopathological study of cyclophosphamide-induced hemorrhagic cystitis - comparison of the effects of dexamethasone and Mesna
}

\section{M.M. Morais ${ }^{1}$ \\ J.N. Belarmino-Filho', G.A.C. Brito ${ }^{2}$ and R.A. Ribeiro ${ }^{1}$}

\author{
Departamentos de ${ }^{1}$ Fisiologia e Farmacologia, and \\ ${ }^{2}$ M orfologia, Faculdade de Medicina, Universidade Federal do Ceará, \\ Fortaleza, CE, Brasil
}

\section{Correspondence \\ R.A. Ribeiro \\ Departamento de Fisiologia e \\ Farmacologia \\ Faculdade de Medicina, UFC \\ Rua Cel. Nunes de Melo, 1127 \\ 60430-270 Fortaleza, CE \\ Brasil \\ Fax: + 55-85-243-9333 \\ E-mail: gerly@ fortalnet.com.br}

Received April 13, 1999

Accepted August 11, 1999

\section{Abstract}

Chemotherapy with oxazaphosphorines, such as cyclophosphamide (CYP), is often limited by unacceptable urotoxicity. Without uroprotection, hemorrhagic cystitis (HC) becomes dose-limiting. To compare the uroprotective efficacy of classical 2-mercaptoethanesulfonic acid (Mesna) treatment with dexamethasone in CYP-induced HC, male Wistar rats (150-200 g; $\mathrm{N}=6$ in each group) were treated with saline or Mesna (40 mg/kg, ip) immediately and 4 and $8 \mathrm{~h}$ after ip administration of CYP $(200 \mathrm{mg} / \mathrm{kg})$. One, 2 or 3 doses of Mesna were replaced with dexamethasone $(1 \mathrm{mg} / \mathrm{kg}$, ip $)$. The animals were sacrificed $24 \mathrm{~h}$ later. Cystitis was evaluated by determining the changes in bladder wet weight (BWW) and by macroscopic and microscopic analysis. CYP treatment induced a marked increased in BWW (162\%, $\mathrm{P}<0.05)$, which was significantly inhibited by treatment with 3 doses of Mesna $(\mathrm{P}<0.05 ; 80 \%)$. The replacement of 1 or 2 doses of Mesna with dexamethasone reduced the increase in BWW by 83.3 and $95 \%$, respectively. Macroscopic analysis of the bladder of rats with CYPinduced $\mathrm{HC}$ showed severe edema and hemorrhage, confirmed by microscopic analysis, that also showed mucosal erosion, inflammatory cell infiltration and ulcerations. The replacement of 1 or 2 doses of Mesna with dexamethasone inhibited the CYP-induced increase in BWW and almost abolished the macroscopic and microscopic alterations, with no significant difference between the effects of Mesna and dexamethasone, indicating that both drugs were efficient in blocking HC. However, although the replacement of all Mesna doses with dexamethasone reduced the edema, it did not prevent $\mathrm{HC}$, suggesting that Mesna is necessary for the initial uroprotection.

\section{Key words}

- Cyclophosphamide

- Hemorrhagic cystitis

- Dexamethasone

- Mesna
Cyclophosphamide (CYP), a cytotoxic alkylating agent, is used in chemotherapeutic regimens of lymphoproliferative disorders, certain solid tumors, and non-neoplastic diseases such as nephrotic syndrome, systemic lupus erythematosus and rheumatoid arthritis (1). Hemorrhagic cystitis is a known adverse effect of this drug and may be the limiting factor in its use $(2,3)$. In the absence of adequate uroprotection, the incidence of this side effect of CYP therapy varies from 2 to $40 \%$ in patients taking low doses of CYP 
on a long-term basis (4). Mortality has been reported to be 2 to $4 \%$ in patients with massive hemorrhage, who were treated with a high dose of intravenous CYP (5). The cause of bladder damage appears to be related to acrolein, a urotoxic metabolite of CYP. It has been proposed that urothelial damage occurs by direct contact with acrolein, which causes edema, ulceration, neovascularization, hemorrhage, and necrosis (6).

Mesna (2-mercaptoethanesulfonic acid), a thiol compound, entered clinical trials as a systemic uroprotective agent in the late 1970s, becoming the drug of choice for this purpose within a short period of time (7). The administration of systemic Mesna results in regional detoxication of the urinary system $(8,9)$. The interaction between acrolein and Mesna results in an inactive compound (10). Thus, Mesna is indicated to prevent the occurrence of hemorrhagic cystitis, but is not effective when the lesion has been established.

Over the last few years, our laboratory has been dedicated to the study of inflammatory mediators involved in the pathogenesis of experimental cyclophosphamide and ifosfamideinduced hemorrhagic cystitis. We demonstrated that cytokines like TNF- $\alpha$ and IL-1 were crucial mediators involved in these inflammatory events and also in urothelial damage and hemorrhage (11). An important contribution to this issue by our group was our recent demonstration that nitric oxide (NO) is the final mediator of urothelial damage and hemorrhage in cystitis (12). In addition, we have demonstrated that the administration of whole anti-TNF- $\alpha$ serum significantly decreased the CYP-induced vesical edema as well as the rise in inducible NOS activity. The induction of NOS in the inflamed bladder appears to require the action of TNF- $\alpha$ and platelet-activating factor (PAF) $(11,12)$.

Glucocorticoids are potent inhibitors of the synthesis of cytokines like TNF- $\alpha$ and IL1, PAF and NOS-inducible expression (13). Thus, the aim of the present study was to investigate the effects of dexamethasone in
CYP-induced hemorrhagic cystitis and thus propose an alternative posologic regimen of lower cost compared to the use of Mesna in the prevention of this type of cystitis.

Six groups of six male Wistar rats weighing 150-200 g were used in the experiments. The animals were kept in appropriate boxes with water and food ad libitum. The animals received an ip injection of saline or CYP (Genuxal $^{\circledR}, 200 \mathrm{mg} / \mathrm{kg}$; Asta Medica, AG Frankfurt, Germany) and were then treated with saline or Mesna (Mitexan ${ }^{\circledR} 200,40 \mathrm{mg}$ / $\mathrm{kg}$, ip; Asta Medica) at the same time and 4 and $8 \mathrm{~h}$ after CYP. In the other experimental groups, 1, 2 or 3 doses of Mesna were replaced with dexamethasone (Decadron ${ }^{\circledR}, 1$ $\mathrm{mg} / \mathrm{kg}$, ip; Prodrome Química \& Farmacêutica Ltda., São Paulo, SP, Brazil). The animals were sacrificed $24 \mathrm{~h}$ after the administration of cyclophosphamide and their bladders were removed by careful dissection.

Bladders were excised, freed from surrounding connective tissue, and examined grossly for edema and hemorrhage. Histological examination was performed by a pathologist in a single-blind fashion. Edema, bleeding, and histological changes were evaluated according to the criteria of Gray et al. (14) as follows: edema was considered severe $(3+)$ when fluid was seen externally and internally on the walls of the bladder, moderate $(2+)$ when confined to the internal mucosa, mild $(1+)$ when normal to moderate, and absent $(0)$. Hemorrhage was scored as follows: $3+$, intravesical clots; $2+$, mucosal hematomas; $1+$, telangiectasia or dilatation of the bladder vessels; 0 , normal. Histopathology was scored as follows: 0 , normal epithelium and absence of inflammatory cell infiltration and ulceration; 1, mild changes involving reduction of epithelial cells, flattening with submucosal edema, mild hemorrhage, and few ulcerations; 2, severe changes including mucosal erosion, inflammatory cell infiltration, fibrin deposition, hemorrhage, and multiple ulcerations.

Bladder wet weight (reported as the mean 
\pm SEM/100 g body weight) and macroscopic and microscopic observations (reported as medians and range) of the bladders were used to evaluate the effects of the drugs. For the analysis of bladder wet weight, data were assessed by analysis of variance (ANOVA) followed by Bonferroni's test. For macroscopic and microscopic analysis, we used the Kruskal Wallis and Mann-Whitney tests to compare medians. $\mathrm{P}<0.05$ was considered significant.

Intraperitoneal injection of CYP $(200 \mathrm{mg} /$ $\mathrm{kg}$ ) induced a marked increase in bladder wet weight $24 \mathrm{~h}$ after its administration $(162 \%$ compared to control group, $\mathrm{P}<0.05)$. CYPevoked increase in bladder wet weight was significantly inhibited by treatment of rats with 3 doses of Mesna ( $80 \%$ reduction), as well as by the replacement of 1 or 2 doses of Mesna with dexamethasone (83.3 and 95\% reduction, respectively) (Figure 1). There was no significant difference between the effects of these 3 different approaches. However, the replacement of all Mesna doses with dexamethasone did not prevent the increase in bladder wet weight.

Cystitis observed $24 \mathrm{~h}$ after CYP administration was characterized macroscopically by the presence of severe edema, receiving a score of 3 (2-3), and by marked hemorrhage with mucosal hematomas and intravesical clots, receiving a score of 3 (2-3), being significantly $(\mathrm{P}<0.05)$ different from the control group which received a score of $0(0-0)$ for edema and hemorrhage. Treatment with Mesna alone or in combination with 1 or 2 doses of dexamethasone, but not dexamethasone alone, significantly reduced the intensity of cystitis $(\mathrm{P}<0.05)$, as indicated by the scores in Table 1.

According to Gray's histopathological criteria, $24 \mathrm{~h}$ after CYP administration there was histological evidence of cystitis: extensive mucosal erosion with ulceration, fibrin deposition, hemorrhage, edema, and leukocyte infiltration, receiving a score of 2 (2-2) (Figure 2B). These alterations were almost abolished $(\mathrm{P}<0.05)$ by the replacement of 1 or 2 doses of Mesna with dexamethasone in the CYP-induced hemorrhagic cystitis, with results similar to those obtained with 3 doses of Mesna (Figure 2C and D). The scores of Gray's parameters (median and range) are shown in Table 1.

In the present study we present evidence

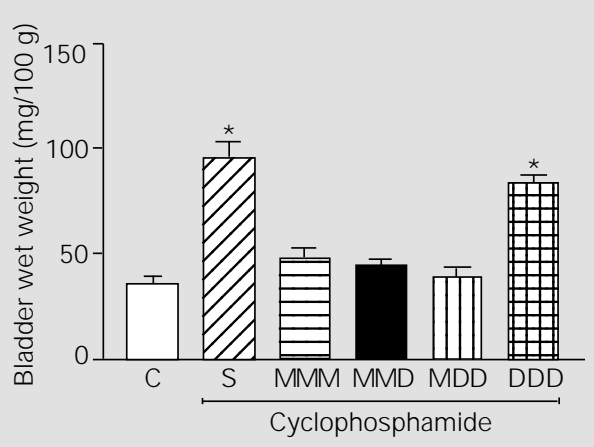

Figure 1 - Effect of different treatments of dexamethasone on bladder wet weight in cyclophosphamide-induced hemorrhagic cystitis. Cyclophosphamide $(200 \mathrm{mg} / \mathrm{kg})$-induced increase in bladder wet weight was measured $24 \mathrm{~h}$ after the induction of cystitis in vehicle (saline, S)-, or Mesna-treated animals (MMM - 3 doses of Mesna at 5 min before and 4 and $8 \mathrm{~h}$ after), Mesna + dexamethasone-treated animals (MMD - 2 doses of Mesna at 5 min before and $4 \mathrm{~h}$ after and 1 dose of dexamethasone at $8 \mathrm{~h}$ after; MDD - 1 dose of Mesna at 5 min before and 2 doses of dexamethasone 4 and $8 \mathrm{~h}$ after) and dexamethasone-treated animals (DDD - 3 doses of dexamethasone at $5 \mathrm{~min}$ before and 4 and $8 \mathrm{~h}$ after). The results are reported as means \pm SEM $(N=6)$. $* P<0.05$ compared to control group $(C$, treated with saline alone) by ANOVA and Bonferroni's tests.

Table 1 - Macroscopic and microscopic analysis of the effects of dexamethasone in cyclophosphamide-induced hemorrhagic cystitis.

Cyclophosphamide (200 mg/kg, ip)-induced macroscopic and microscopic alterations were evaluated $24 \mathrm{~h}$ after its administration in vehicle (saline, S)-, or Mesna-treated animals (MMM - 3 doses of Mesna at 5 min before and 4 and $8 \mathrm{~h}$ after), Mesna + dexamethasone-treated animals (MMD - 2 doses of Mesna at 5 min before and $4 \mathrm{~h}$ after and 1 dose of dexamethasone at $8 \mathrm{~h}$ after; MDD - 1 dose of Mesna at $5 \mathrm{~min}$ before and 2 doses of dexamethasone 4 and $8 \mathrm{~h}$ after) and dexamethasone-treated animals (DDD - 3 doses of dexamethasone at $5 \mathrm{~min}$ before and 4 and $8 \mathrm{~h}$ after). The results are reported as median and range $(\mathrm{N}=6)$. $* \mathrm{P}<0.05$ compared to the control group ( $\mathrm{C}$, treated with saline alone) by Kruskal Wallis and Mann-Whitney tests.

\begin{tabular}{lccl}
\hline Groups & $\begin{array}{c}\text { Macroscopic analysis } \\
\text { (edema) }\end{array}$ & $\begin{array}{c}\text { Macroscopic analysis } \\
\text { (hemorrhage) }\end{array}$ & Microscopic analysis \\
\hline C & $0(0-0)$ & $0(0-0)$ & $0(0-0)$ \\
S & $3(2-3)^{*}$ & $3(2-3)^{*}$ & $2(2-2)^{*}$ \\
MMM & $0(0-1)$ & $0(0-2)$ & $0.5(0-1)$ \\
MMD & $0(0-0)$ & $0(0-0)$ & $0(0-1)$ \\
MDD & $0(0-0)$ & $0(0-0)$ & $0(0-0)$ \\
DDD & $1(0-3)^{*}$ & $1(1-3)^{*}$ & $2(1-2)^{*}$
\end{tabular}


Figure 2 - Histological analysis of representative bladder walls in cross section. A, Normal bladder. B, Cystitis in a rat treated $24 \mathrm{~h}$ previously with cyclophosphamide (200 $\mathrm{mg} / \mathrm{kg}$, ip). Intense urothelial damage, edema, leukocyte infiltration, hemorrhage and fibrin deposition. C, Bladder of rats treated with 3 doses of Mesna ( $20 \%$ of cyclophosphamide dose, $40 \mathrm{mg} / \mathrm{kg}$, ip). Urothelium preservation and absence of edema, hemorrhage, leukocyte infiltration and ulcerations. D, Bladder of rats treated with 1 dose of Mesna and 2 doses of dexamethasone (1 mg/kg, ip). Absence of microscopic alterations, comparable to the treatment with 3 doses of Mesna. Hematoxylin-eosin, X40. Bar, $250 \mu \mathrm{m}$.
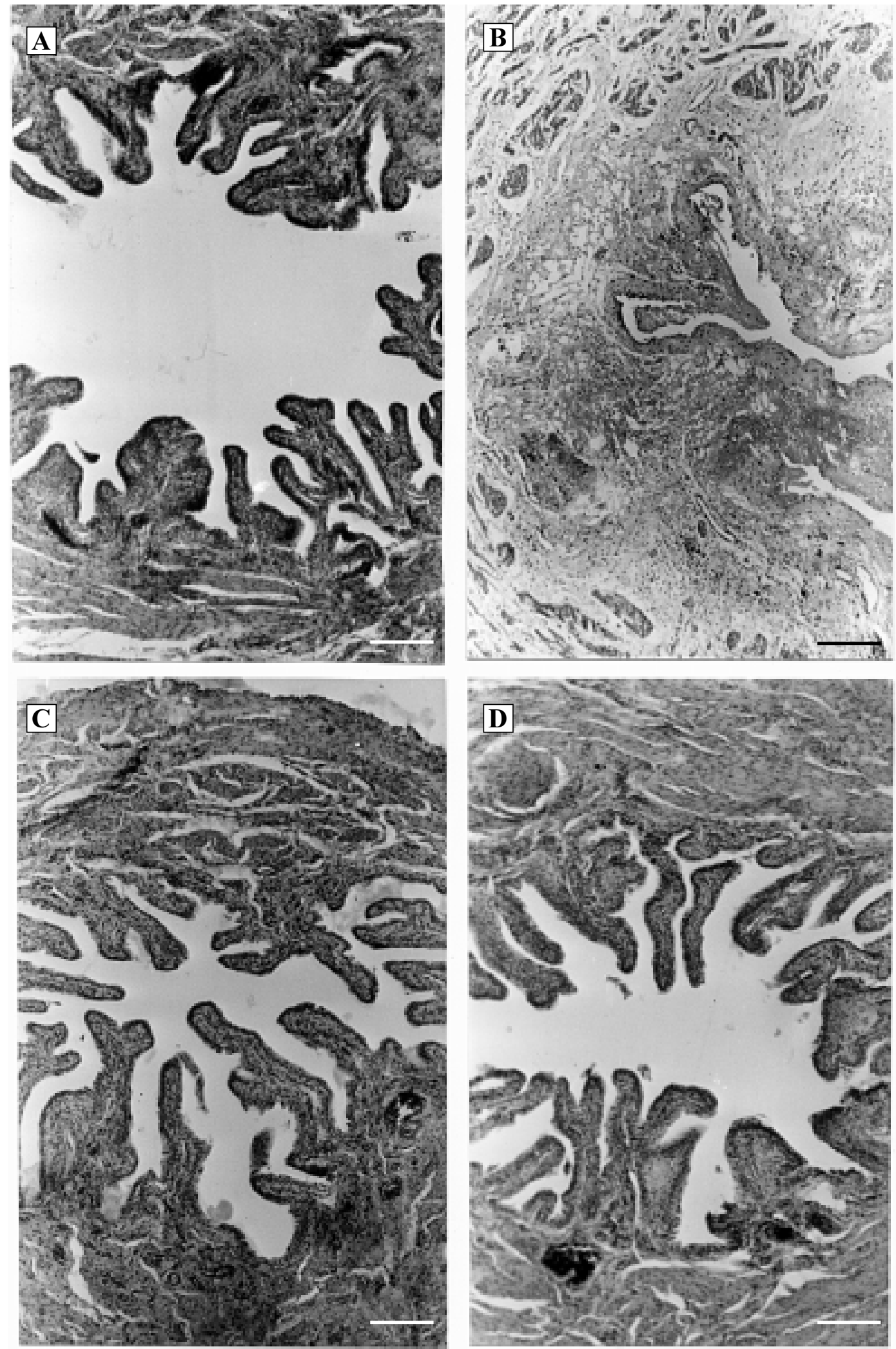
indicating that dexamethasone could be a useful tool when combined with Mesna in the preventive management ofCYP-induced hemorrhagic cystitis. In agreement with these data, it was recently reported that patients using corticosteroid as an immunosuppressor, combined with cyclophosphamide in the treatment of systemic lupus erythematosus, have a low incidence of hemorrhagic cystitis (15).

Previous data from our laboratory have demonstrated that cytokines like TNF- $\alpha$, IL$1, \mathrm{NO}$ and PAF were crucial mediators involved in these inflammatory events and also in the urothelial damage and hemorrhage. Thus, the present results showing that dexamethasone inhibits CYP-induced hemorrhagic cystitis could be explained by potent effect of the corticoid as an inhibitor of the synthesis of cytokines like TNF- $\alpha$ and
IL-1, PAF and of NOS-inducible expression. It is important to note that Mesna seems to be necessary for the initial uroprotection through its neutralizing effect on urothelial damage initiated by acrolein, while dexamethasone may be inhibiting the mediators of the inflammatory process that follows.

The advantage of this alternative posologic regimen is lower cost and the fact that dexamethasone is already routinely used as a standard component of antiemetic regimens for numerous cancer chemotherapy protocols (16) and as an immunosuppressor in autoimmune diseases like systemic lupus erythematosus (15). Thus, dexamethasone could be tested in clinical trials as a substitute of the two last doses of Mesna for the inhibition of CYPinduced hemorrhagic cystitis.

\section{References}

1. Calabresi P \& Chabner BA (1992). Antineoplasic agents. In: Gilman AF, Rall TW, Niss AS \& Taylor $P$ (Editors), The Pharmacological Basis of Therapeutics. 8th edn. McGraw-Hill, Singapore.

2. Stillwell TJ \& Benson RC (1988). Cyclophosphamide-induced hemorrhagic cystitis - A review of 100 patients. Cancer, 61 : 451-457.

3. Philips FS, Sternberg SS, Cronin AP \& Vidal PM (1961). Cyclophosphamide and urinary bladder toxicity. Cancer Research, 21: 1577-1589.

4. Foad BSI \& Hess EV (1976). Urinary bladder complications with cyclophosphamide therapy. Archives of Internal Medicine, 136: 616-625.

5. Etlik O, Tomur A, Deveci S, Piskin I \& Pekcan M (1997). Comparison of the uroprotective efficacy of Mesna and HBO treatments in cyclophosphamide-induced hemorrhagic cystitis. J ournal of Urology, 158: 2296-2299.

6. CoxPJ (1979). Cyclophosphamide cystitis - Identification of acrolein as the causative agent. Biochemical Pharmacology, 28: 2045-2049.

7. Katz A, Epelman S, Anelli A, Gorender EF, Cruz SM, Oliveira RM \& Marques LA (1995). A prospective randomized evaluation of three schedules of Mesna admin- istration in patients receiving an ifosfamide-containing chemotherapy regimen: sustained efficiency and simplified administration. J ournal of Cancer Research and Clinical Oncology, 121: 128-131.

8. Elias AD, Eder J P, Shea T, Begg CB, Frei 3rd E \& Antman KH (1990). High dose ifosfamide with Mesna uroprotection: a phase I study. J ournal of Clinical Oncology, 8: 170-178.

9. Scheulen ME, Niederle N, Bremer K, Schutte J \& Seeber S (1983). Efficacy of ifosfamide in refractory malignant diseases and uroprotection by Mesna: results of a clinical phase II study with 151 patients. Cancer Treatment Reviews, 10: 93-101.

10. Wagner T (1994). Ifosfamide clinical pharmacokinetics. Clinical Pharmacokinetics, 26: 439-456.

11. Gomes TNA, Santos CC, Souza-Filho MVP, Cunha FQ \& Ribeiro RA (1995). Participation of TNF- $\alpha$ and IL-1 in the pathogenesis of cyclophosphamide-induced hemorrhagic cystitis. Brazilian J ournal of Medical and Biological Research, 28: 1103-1108.

12. Souza-Filho MVP, Lima MVA, Pompeu MML, Ballejo G, Cunha FQ \& Ribeiro RA (1997). Involvement of nitric oxide in the pathogenesis of cyclophosphamide-in- duced hemorrhagic cystitis. American J ournal of Pathology, 150: 247-256.

13. Ribeiro RA, Souza-Filho MVP, Santos CC, Lima MVA, Pompeu MML, Ballejo G \& Cunha FQ (1998). Involvement of nitric oxide and tumor necrosis factor in the pathogenesis of cyclophosphamide-induced hemorrhagic cystitis. In: Moraes M, Brentani R \& Bevilacqua R (Editors), Proceedings of the17th International Cancer Congress. Monduzzi Editore, Bologna, Italy, 227-231.

14. Gray $\mathrm{KJ}$, Engelmann UH, J ohnson EH \& Fishman IJ (1986). Evaluation of misoprostol cytoprotection of the bladder with cyclophosphamide (cytoxan) therapy. J ournal of Urology, 133: 497-500.

15. Rocha FAC \& Oliveira KRB (1997). Cyclophosphamide-induced hemorrhagic cystitis in SLE. Revista Brasileira de Reumatologia, 37: 89-92.

16. Hesketh PJ, Harvey WH, Harker WG, Beck TM, Ryan T, Bricker IJ, Kish J A, Murphy WK, Hainsworth J D \& Haley B (1994). A randomized double-blind comparison of intravenous ondansetron alone and in combination with intravenous dexamethasone in the prevention of high-dose cisplatin-induced emesis. J ournal of Clinical Oncology, 12: 596-600. 